\title{
Irisfixierte phake Intraokularlinse
}

\author{
Marie Dietrich · Sarah Moussa - Josef Ruckhofer - Esther Halbwirth · Herbert Reitsamer
}

Eingegangen: 14. Mai 2019 / Angenommen: 29. Juli 2019 / Online publiziert: 22. August 2019

(C) Der/die Autor(en) 2019

\section{Zusammenfassung}

Hintergrund In der refraktiven Chirurgie können wir zwischen zahlreichen Verfahren zur Behandlung von Fehlsichtigkeiten auswählen. Im Allgemeinen unterscheidet man zwischen additiven Verfahren, bei denen dem Patienten eine künstliche Linse implantiert wird (refraktive Linsenimplantation), und subtraktiven Verfahren, bei denen dem Patienten Gewebe der Hornhaut mittels unterschiedlichen Lasermethoden abgetragen wird. Im folgenden Artikel möchten wir speziell auf die irisfixierte phake Vorderkammerlinse eingehen.

Material und Methode Derzeit gibt es starre und flexible irisfixierte Vorderkammerlinsen. Der große Vorteil der faltbaren Version gegenüber der starren Linse besteht darin, dass die gefaltete Linse über einen nur 3,2 mm kleinen Schnitt ins Auge implantiert werden kann und somit der durch die Operation induzierte Astigmatismus vermindert und die Heilungsdauer verkürzt wird.

Resultate Bei 136 Augen von 79 Patienten im mittleren Alter von $38 \pm 9,5$ Jahren (20 bis 61 Jahre) mit einem mittleren sphärischen Äquivalent von $-8,85 \mathrm{dpt} \pm 2,28 \mathrm{dpt}(-2,63$ bis $-13,75 \mathrm{dpt})$ und einem mittleren refraktiven Astigmatismus von $1,95 \mathrm{dpt} \pm$ $0,82 \mathrm{dpt}$ ( 1 bis $5 \mathrm{dpt}$ ) wurde eine torische Artiflex ${ }^{\circledR}$ implantiert. Nach 24 Monaten liegt das mittlere sphärische Äquivalent bei $-0,08 \mathrm{dpt}(-1,25$ bis $+0,63 \mathrm{dpt})$, $100 \%$ liegen innerhalb von $\pm 1 \mathrm{dpt}, 96 \%$ innerhalb von $\pm 0,5 \mathrm{dpt}$ der gewünschten Zielrefraktion. Zwei Jahre postoperativ erreichen $85 \%$ der Augen einen unkorrigierten Fernvisus von 1,0 oder besser, 54\% von

\footnotetext{
M. Dietrich $(\varangle) \cdot$ S. Moussa · J. Ruckhofer · E. Halbwirth ·

H. Reitsamer

Universitätsklinik für Augenheilkunde und Optometrie,

Paracelsus Medizinische Privatuniversität, Müllner

Hauptstraße 48, 5020 Salzburg, Österreich

m.dietrich@salk.at
}

1,25 oder besser und $16 \%$ von 1,6 oder besser. Der Endothelzellverlust beträgt 0,89\%. Es kam zu keinen postoperativen Komplikationen.

Schlussfolgerung Die Implantation von irisfixierten phaken Intraokularlinsen ist ein sichereres, effizientes, vorhersagbares und stabiles Verfahren zur Korrektur von moderater und hoher Myopie und Astigmatismus.

Schlüsselwörter Refraktive Chirurgie $\cdot$ Artisan · Artiflex $\cdot$ Veriflex $\cdot$ Myoper Astigmatismus

\section{Iris claw phakic intraocular lens}

\section{Summary}

Background In refractive surgery there is a choice between numerous procedures for the treatment of refractive errors. In general, there are additive methods in which an artificial lens is implanted (refractive lens implantation) and subtractive methods in which corneal tissue is removed using different laser methods. This article focuses on the iris claw phakic intraocular lens.

Material and methods Currently, there are rigid and flexible iris claw phakic intraocular lenses available. The great advantage of the foldable version compared to the rigid lens is that the flexible lens can be implanted into the eye through a small $3.2 \mathrm{~mm}$ incision, resulting in decreased surgically induced astigmatism and faster visual recovery

Results In this study 136 eyes from 79 patients with a mean age of $38 \pm 9.5$ years (range 20-61 years), a mean spherical equivalent of $-8.85 \mathrm{D} \pm 2.28 \mathrm{D}$ (range $-2.63 \mathrm{D}$ to $-13.75 \mathrm{D}$ ) and a mean preoperative cylinder of $1.95 \mathrm{D} \pm 0.82 \mathrm{D}(1-5 \mathrm{D})$ underwent implantation of toric Artiflex ${ }^{\circledR}$ phakic intraocular lens. After 24 months the mean spherical equivalent was $-0.08 \mathrm{D}(-1.25 \mathrm{D}$ to $+0.63 \mathrm{D}$ ), all eyes were within $\pm 1 \mathrm{D}$ and $96 \%$ of the eyes were within $\pm 0.5 \mathrm{D}$ of the target refraction. The 
uncorrected distance visual acuity (UDVA) was 1.0 or better in $85 \%, 1.25$ or better in $54 \%$ and 1.6 or better in $16 \%$ of eyes 2 years after surgery. A slight loss of endothelial cells of $0.89 \%$ occurred at 24 months. There were no postoperative complications.

Conclusion The implantation of iris claw phakic intraocular lenses is an effective, predictable, stable, and safe method for the correction of moderate and high myopia and astigmatism.

Keywords Refractive surgery · Artisan · Artiflex · Veriflex $\cdot$ Myopic astigmatism

\section{Hintergrund}

In den letzten Jahrzehnten hat sich die LASIK (Laser-in-situ-Keratomileusis) bzw. Femto-LASIK als Methode der Wahl zur Behandlung von Fehlsichtigkeiten durchgesetzt und wird weltweit am häufigsten durchgeführt. Als Alternative gibt es andere keratorefraktive Behandlungsmöglichkeiten, wie z.B. PRK (Photorefraktive Keratektomie) oder SMILE (Small Incision Lenticule Extraction).

Jedoch können nicht alle Sehfehler mit laserchirurgischen Verfahren behandelt werden. Gerade bei höherer Kurz- oder Weitsichtigkeit bzw. bei dünnen Hornhäuten sind phake Intraokularlinsen (pIOL) eine sichere und effektive Behandlungsmethode.

Ein weiterer wesentlicher Vorteil von pIOLs ist die Reversibilität und die Beibehaltung der ursprünglichen Kurvatur der Hornhaut - v. a. im Hinblick auf die Biometrie bei einer allfälligen späteren Kataraktentwicklung.

Zurzeit gibt es 2 verschiedene Typen von pIOLs: irisfixierte pIOLs und phake Hinterkammerlinsen (EVO Visian ICL, Staar Surgical und Implantable Phakic Contact Lens IPCL, Polytech Domilens).

Wir möchten in diesem Artikel einen Überblick über die irisfixierten pIOLs geben.

Barraquer, Strampelli, Dannheim und Choyce leisteten in den 50er-Jahren die Pionierarbeit in der Entwicklung von - damals kammerwinkelgestützten Vorderkammerlinsen. Aufgrund der zahlreichen Komplikationen wie die postoperative Entstehung von Hornhautdekompensationen oder Glaukom konnte sich diese Methode jedoch nicht durchsetzen [1].

Im Jahr 1977 hatte Jan Worst die Idee, IOLs an der Iris zu fixieren. Diese Linsen aus Polymethylmethacrylat (PMMA) wurden zunächst zur Behandlung der Aphakie eingesetzt.

Im Jahr 1986 implantierten Fechner und Worst die erste irisfixierte IOL (Artisan ${ }^{\circledR}$, Ophtec BV) in ein myopes phakes Auge [2].

Im Jahr 2001 erfolgte die Einführung der torischen Artisan $^{\circledR}$ (Ophtec BV) [3].

Im Jahr 2005 wurde schließlich eine flexible Version der Artisan ${ }^{\circledR}$-pIOL entwickelt: Die Artiflex ${ }^{\circledR}$-pIOL (Ophtec BV) besteht aus einer Silikonoptik aus Polysiloxane sowie Haptiken aus PMMA [4, 5].
Die torische Version der Artiflex ${ }^{\circledR}$ wurde im September 2009 auf den Markt gebracht, Abb. 1.

Durch diese stetige Entwicklung der Linsenmodelle hat sich die Implantation von irisfixierten pIOLs als eine sehr effektive, sichere, vorhersagbare und stabile Methode zur Korrektur von refraktiven Fehlern herausgestellt.

\section{Material und Methode}

Die Irisfixation wird durch die Form der Linse, einer 5- bzw. 6-mm-Optik mit 2 diametral angeordneten „Klauen“, (Gesamtbreite 8,5 mm) ermöglicht. Derzeit gibt es starre irisfixierte Vorderkammerlinsen aus PMMA (Artisan ${ }^{\circledR}$, Ophtec BV) bzw. die flexible Version aus Polysiloxan mit PMMA-Haptiken (Artiflex ${ }^{\circledR}$, Ophtec BV). Diese Linsen werden auch unter dem Namen Verisyse ${ }^{\circledR}$ bzw. Veriflex ${ }^{\circledR}$ (Johnson \& Johnson Vision) vertrieben. Die jeweils erhältlichen Stärken sind in Tab. 1 aufgelistet.

Der große Vorteil der faltbaren Version (Artiflex ${ }^{\circledR /}$ Veriflex $\left.{ }^{\circledR}\right)$ gegenüber den PMMA-Linsen besteht darin, dass die gefaltete Linse über einen nur $3,2 \mathrm{~mm}$ kleinen Schnitt (anstatt 5,5 mm bzw. 6,5 $\mathrm{mm}$ bei der Artisan ${ }^{\circledR}$ - bzw. Verisyse ${ }^{\circledR}$-Linse) ins Auge implantiert wird. Dadurch wird die Wahrscheinlichkeit eines durch die Operation induzierten Astigmatismus vermindert, und dies führt wiederum zu einer rascheren visuellen Genesung [4-6].

\section{Operationstechnik}

Bei Kontaktlinsenträgern wird eine Karenzzeit von mindestens 1 Woche (bei weichen) bzw. 1 Monat (bei harten Kontaktlinsen) für die Linsenberechnung empfohlen. Zur Vermeidung eines postoperativen $\mathrm{Pu}-$ pillarblocks ist eine Iridotomie mittels YAG-Laser präoperativ bzw. Iridektomie intraoperativ notwendig. Die Operation kann in Sedoanalgesie (bei Wunsch auch in Allgemeinanästhesie) durchgeführt werden und erfolgt nach den Standards der modernen refraktiven Chirurgie.

Für die Implantation einer Artiflex ${ }^{\circledR} /$ Veriflex $^{\circledR}$ wird ein 3,2 mm korneoskleraler Tunnelschnitt bei $12 \mathrm{Uhr}$ vorgenommen und 2 Inzisionen bei 10 und 2 Uhr angelegt.

Die pIOL wird unter Instillation von einem Viskoelastikum (Healon GV®, Johnson \& Johnson Vision) implantiert und vor der Pupille und der Iris zentriert. Dann wird Irisgewebe zwischen den Klauen der Linse enklaviert, sodass diese in der richtigen Position bleibt. Bei torischen Linsen wurde unmittelbar präoperativ noch die Achse, in der die Linse fixiert werden soll, markiert.

Am Ende der Operation wird das Viskoelastikum wieder abgesaugt und ausgespült sowie intrakameral Cefuroxim verabreicht, die Inzision durch Hydration geschlossen sowie die Bindehaut gekautert. 


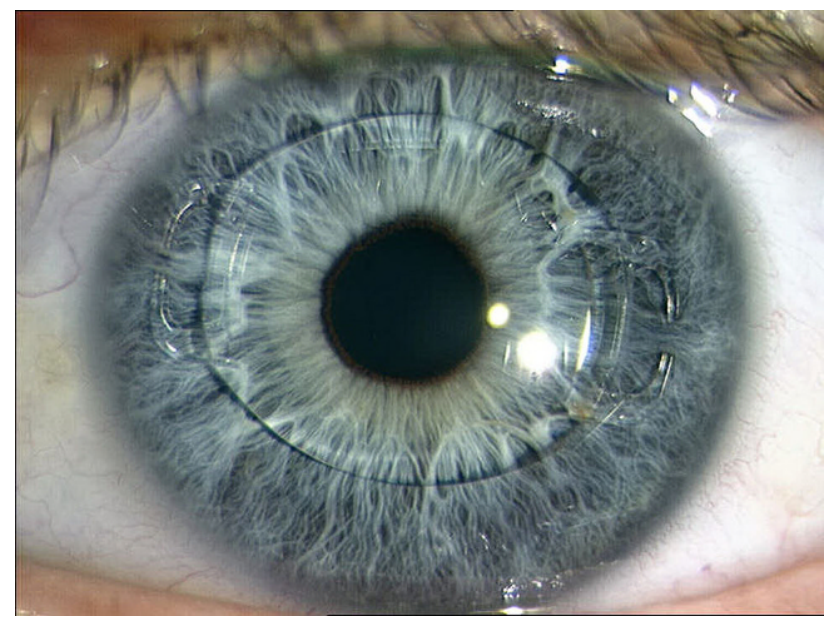

Abb. 1 Torische Artiflex ${ }^{\circledR}$ (Ophtec BV, Groningen, Niederlande)

Die postoperative Therapie beinhaltet: Ofloxacin 0,3\% AT (Ofloxavision sine ${ }^{\circledR}$, Omnivision) 3-mal täglich für 2 Wochen und Prednisolon $1 \%$ AT (Prednifluid $^{\circledR}$, Dermapharm) 5-mal täglich über 5 Wochen ausschleichend.

\section{Einschlusskriterien}

- Alter >18 Jahre

- Stabile Refraktion für mindestens 2 Jahre

- Pupillenweite idealerweise in Dämmerung $<7 \mathrm{~mm}$

- Vorderkammertiefe $>3,2 \mathrm{~mm}$ (gemessen vom Epithel)

- Endothelzellzahl $>2000$ Zellen $/ \mathrm{mm}^{2}$ bzw. altersentsprechend

\section{Ausschlusskriterien}

- Augenerkrankungen wie Glaukom, Katarakt, Uveitis und Retinopathien

- Systemische Erkrankungen (Autoimmunerkrankungen, Diabetes mellitus)

\section{Resultate}

In einer Studie von Dick et al. [7] aus dem Jahr 2009 wurde bei 290 myopen Augen von 191 Patienten ei- ne Artiflex-pIOL implantiert. Alle Patienten waren im Alter von 18 bis 60 Jahren, und das präoperative sphärische Äquivalent lag bei $-7,33 \mathrm{dpt} \pm 2,60 \mathrm{dpt}(-1,5$ bis $-12,63 \mathrm{dpt}$ ). Nach einem Beobachtungszeitraum von 2 Jahren erreichten 97,2\% der Patienten einen unkorrigierten Fernvisus von 0,5 oder besser; $75,2 \%$ der Patienten lagen innerhalb von $0,5 \mathrm{dpt}$ der gewünschten Zielrefraktion, und der Endothelzellverlust betrug nach 2 Jahren $1,07 \%$.

Ruckhofer et al. [8] veröffentlichten 2012 die Daten von 42 myopen Augen (24 Patienten, mittleres Alter 35 Jahre 18 bis 52 Jahre), welche eine torische Artiflex ${ }^{\circledR}$ implantiert bekommen haben. Das präoperative sphärische Äquivalent lag bei $-7,52 \mathrm{dpt} \pm 2,22 \mathrm{dpt}$ $(-2,63$ bis $-13,00 \mathrm{dpt})$ und der mittlere refraktive Astigmatismus bei $-1,82 \mathrm{dpt} \pm 0,96 \mathrm{dpt}(-1$ bis $-5,0 \mathrm{dpt})$. Sechs Monate postoperativ lag das mittlere sphärische Äquivalent bei $-0,05 \mathrm{dpt} \pm 0,20 \mathrm{dpt}$ und der mittlere refraktive Astigmatismus bei $-0,18 \mathrm{dpt} \pm 0,30 \mathrm{dpt}$; $100 \%$ der Augen lagen innerhalb $\pm 0,5 \mathrm{dpt}$ und $86 \%$ innerhalb von $\pm 0,25 \mathrm{dpt}$ der Zielrefraktion. Das mittlere sphärische Äquivalent war bei $-0,05 \mathrm{dpt} \pm 0,20 \mathrm{dpt}$. Alle Augen erreichten zu diesem Zeitpunkt einen unkorrigierten Fernvisus von 0,5 oder besser, $98 \%$ erreichten 0,8 oder besser und $90 \% 1,0$ oder besser. Der Endothelzellverlust betrug $0,72 \%$ nach 6 Monaten.

Es liegen jetzt auch eigene Ergebnisse der Implantation der torischen Artiflex ${ }^{\circledR}$ bei myopem Astigmatismus mit einer Nachkontrollzeit von 2 Jahren vor. Bei 136 Augen von 79 Patienten (49 weiblich, 30 männlich) im mittleren Alter von $38 \pm 9,5$ Jahren (20 bis 61 Jahre) wurde eine torische Artiflex ${ }^{\circledR}$ implantiert. Das präoperative sphärische Äquivalent lag bei $-8,85 \mathrm{dpt} \pm 2,28 \mathrm{dpt}(-2,63$ bis $-13,75 \mathrm{dpt})$, der mittlere refraktive Astigmatismus bei $1,95 \mathrm{dpt} \pm 0,82 \mathrm{dpt}$ ( 1 bis $5 \mathrm{dpt}$ ). Zwei Jahre postoperativ erreichen $85 \%$ der Augen einen unkorrigierten Fernvisus von 1,0 oder besser, $54 \%$ von 1,25 oder besser und $16 \%$ von 1,6 oder besser (Abb. 2). Das sphärische Äquivalent der manifesten Refraktion lag zu diesem Zeitpunkt im Mittel bei $-0,08 \mathrm{dpt}(-1,25 \mathrm{bis}+0,63 \mathrm{dpt}), 100 \%$ lagen innerhalb von $\pm 1 \mathrm{dpt}, 96 \%$ innerhalb von $\pm 0,5 \mathrm{dpt}$ (Abb. 3). Der refraktive Astigmatismus war 24 Monate postoperativ im Mittel bei $0,19 \mathrm{dpt} \pm 0,32 \mathrm{dpt}(0$ bis 1,5 dpt). Der Endothelzellverlust betrug 0,89\%.

Tab. 1 Erhältliche Modelle der irisfixierten phaken Vorderkammerlinsen

\begin{tabular}{|l|l|l|l|l|}
\hline Linsenmodell & Material & Linsenstärke (dpt) & Optische Zone (mm) & Gesamtdurchmesser (mm) \\
\hline Artisan Myopia 206/Verisyse & PMMA & $-1,0$ bis $-23,5$ & 5 & 8,5 \\
\hline Artisan Myopia 204/Verisyse & PMMA & $-1,0$ bis $-15,5$ & 6 & 8,5 \\
\hline Artisan Hyperopia 203 & PMMA & $+1,0$ bis $+12,0$ & 5 & 8,5 \\
\hline Artisan Toric & PMMA & Sphäre $-22,0$ bis $+14,0$ & 5 & 8,5 \\
\hline & & Torus $-1,0$ bis $-7,5$ & & 8,5 \\
\hline Artiflex Myopia 401/Neriflex & PMMA-Haptik/Polysiloxanoptik & $-2,0$ bis $-14,5$ & 6 & 8,5 \\
\hline Artiflex Toric & PMMA-Haptik/Polysiloxanoptik & Sphäre $-1,0$ bis $-13,5$ & 6 & \\
& & Torus $-1,0$ bis $-5,0$ & & \\
\end{tabular}




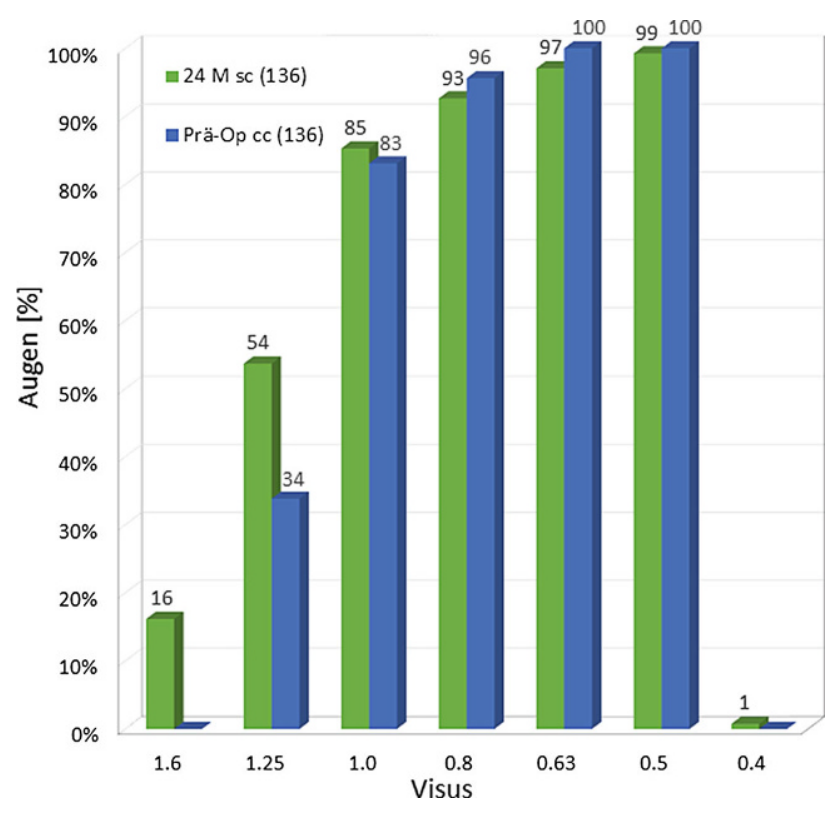

Abb. 2 Vergleich unkorrigierter Fernvisus (sc) 24 Monate postoperativ mit korrigiertem Fernvisus (cc) präoperativ

Postoperativ kam es nach 1 Monat im Mittel zu einer geringen Augendruckerhöhung (15,53 mm Hg vs. $14,18 \mathrm{~mm} \mathrm{Hg}$ präoperativ), bei der Kontrolle nach 2 Jahren lag der Augendruck im Mittel wieder bei $14,81 \mathrm{~mm} \mathrm{Hg}$.

Präzipitate bzw. Makrophagen auf der pIOL traten sowohl kurz postoperativ als auch nach längerer Zeit auf. In den meisten Fällen waren diese aber temporär und mit einer kurzzeitigen Applikation von kortisonhaltigen Augentropfen gut behandelbar.

\section{Diskussion}

In den letzten Jahren konnte in zahlreichen Studien gezeigt werden, dass die Implantation von irisfixierten pIOLs ein sichereres, effizientes und stabiles Verfahren zur Korrektur von Myopie und Astigmatismus darstellt. Alle Studien zeigen eine Verbesserung des postoperativen unkorrigierten Fernvisus [3-7].

Die Implantation von irisfixierten pIOLs hat sich auch durch die genaue Vorhersagbarkeit bewährt. In eigenen Daten beträgt das sphärische Äquivalent der manifesten Refraktion im Mittel $-0,08$ dpt $(-1,25$ bis $+0,63 \mathrm{dpt}), 100 \%$ liegen innerhalb von $\pm 1 \mathrm{dpt}$, $96 \%$ innerhalb von $\pm 0,5 \mathrm{dpt}$ nach 2 Jahren (torische Artiflex $\left.{ }^{\circledR}\right)$. Dies ist vergleichbar mit einer anderen Studie von Dick et al., in der 75,3\% der Augen nach 6 Monaten, 75,5\% nach 1 Jahr und $75,2 \%$ nach 2 Jahren innerhalb von $\pm 0,5 \mathrm{dpt}$ liegen [7].

Stellt man die irisfixierten pIOLs der EVO Visian ICL gegenüber, konnte in mehreren Studien kein signifikanter Unterschied hinsichtlich postoperativem unkorrigiertem und korrigiertem Visus festgestellt werden. Beide Operationsmethoden stellen eine si-

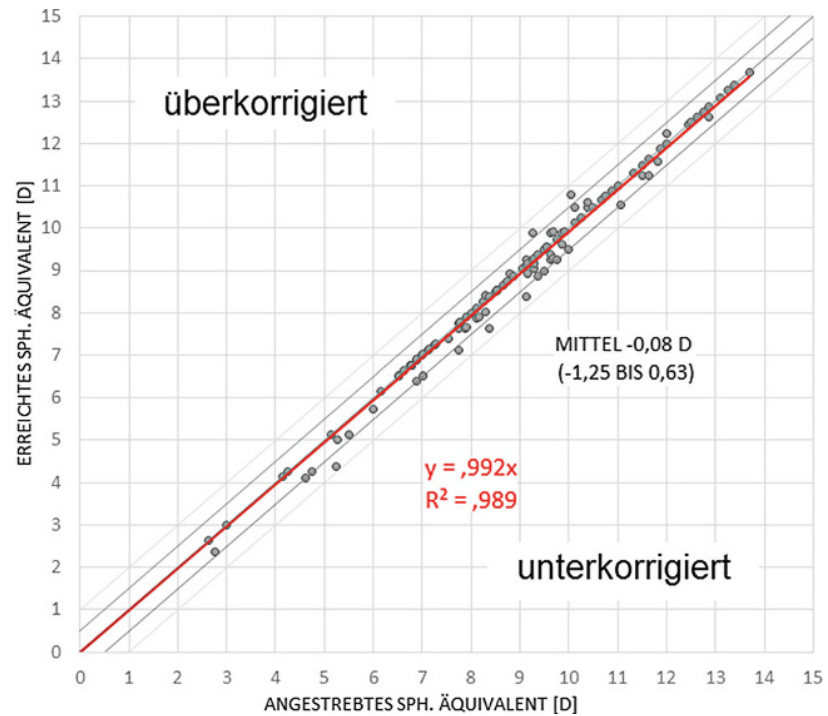

Abb. 3 Angestrebte vs. erreichte Korrektur (sphärisches Äquivalent), 24 Monate postoperativ

chere und effektive Möglichkeit zur Korrektur von hoher Myopie und Astigmatismus dar [9, 10].

Eines der wesentlichen Bedenken bei der Implantation von irisfixierten Vorderkammerlinsen ist der Verlust von Endothelzellen. Der größte Endothelzellverlust ist innerhalb der ersten 6 Monate nach Implantation festzustellen, da die Hauptursache für den Zellverlust die Operation an sich darstellt [11]. Sowohl die Manipulation des Hornhautendothels intraoperativ als auch die postoperative Lageveränderung und subklinische Entzündung können zum Verlust von Endothelzellen führen [6]. Eigene Ergebnisse zeigten lediglich einen Endothelzellverlust von 0,89\% nach 2 Jahren.

In einer Vergleichsstudie konnten Awadein et al. zwar einen höheren Verlust von Endothelzellen bei irisfixierten Vorderkammerlinsen gegenüber einer Evo Visian ICL feststellen, dieser war aber nicht signifikant. Dies deckt sich auch mit einer Studie von Ghoreishi et al. [9, 12].

Die Messung der Vorderkammertiefe $(>3,2 \mathrm{~mm}$ gemessen vom Epithel) und die dem Alter entsprechende Endothelzelldichte sind bei der Patientenauswahl $\mathrm{zu}$ berücksichtigen. Daher ist es auch unerlässlich, den Patienten darüber zu informieren, dass exzessives Augenreiben (z. B. bei Atopikern) nach refraktiver Linsenimplantation zu Schädigungen der Hornhaut führen kann [13].

Vorderkammerlinsen können durch ihre Optik einen Pupillarblock und dadurch ein akutes Glaukom induzieren. Daher ist auch eine präoperative YAG-Iridotomie bzw. intraoperative Iridektomie notwendig. Wir konnten bei einigen Patienten frühpostoperativ eine passagere Augendruckerhöhung feststellen 
(Kortisonresponder!), in keinem Fall wurde aber eine dauerhafte antiglaukomatöse Therapie benötigt.

Da die pIOL direkt im Irisgewebe fixiert wird, kann es durch Augenbewegungen und Augenreiben $\mathrm{zu}$ Scherkräften an der Iris kommen. Das damit verbundene mögliche Auftreten von Pigmentdispersion und chronischen Entzündungen tritt nur in Einzelfällen auf. In einer Studie von Menezo et al. wird hinsichtlich einer Pigmentdispersion von einer Langzeitinzidenz von $6,6 \%$ innerhalb von 10 Jahren berichtet [14].

Die Ovalisierung der Pupille kommt sehr selten vor und hat klinisch keine Relevanz. Die Deenklavation der Haptik kann bei Patienten durch das Ausüben bestimmter Sportarten (Motocross, Bungee-Jumping, Power-Plate, Trampolinspringen) oder auch spontan auftreten. Eine deenklavierte Haptik kann in einer weiteren Operation wieder reenklaviert werden.

Die Ausbildung einer Katarakt als potenzielle Nebenwirkung ist bei Patienten mit irisfixierter Vorderkammerlinse sehr unwahrscheinlich. In einer Studie von Chen et al. konnte bei Patienten, welche mit einer EVO Visian ICL versorgt wurden, eine höhere Inzidenz für die Kataraktentwicklung als bei Patienten mit irisfixierter pIOL festgestellt werden [15].

Das Risiko der Netzhautablösung ist bei myopen Patienten ohnehin gegeben und kann nicht in direkten Zusammenhang mit der Implantation einer pIOL gebracht werden.

\section{Schlussfolgerung}

Die Implantation von irisfixierten phaken Intraokularlinsen stellt ein sichereres, effizientes, vorhersagbares und stabiles Verfahren zur Korrektur von moderater und hoher Myopie und Astigmatismus dar. Es ist eine exzellente refraktive Alternative zu Laserverfahren. Erfolg und Zufriedenheit der Patienten hängen aber wie bei allen refraktiven Eingriffen von einer akkuraten Patientenselektion, ausführlichen Aufklärung, aber auch von der Operationstechnik und der postoperativen Nachsorge ab.

Funding Open access funding provided by Paracelsus Medical University.

Interessenkonflikt M. Dietrich, S. Moussa, J. Ruckhofer, E. Halbwirth und H. Reitsamer geben an, dass kein Interessenkonflikt besteht.

Open Access Dieser Artikel wird unter der Creative Commons Namensnennung 4.0 International Lizenz (http:// creativecommons.org/licenses/by/4.0/deed.de) veröffentlicht, welche die Nutzung, Vervielfältigung, Bearbeitung, Verbreitung und Wiedergabe in jeglichem Medium und Format erlaubt, sofern Sie den/die ursprünglichen Autor(en) und die Quelle ordnungsgemäß nennen, einen Link zur Creative
Commons Lizenz beifügen und angeben, ob Änderungen vorgenommen wurden.

\section{Literatur}

1. Mertens EL, Baikoff G. Phakic IOLS. Where are we now? Cataract Refract Surg Now Today Eur. 2018;November/ December:64-67.

2. Fechner PU, Worst JGF. A new concave intraocular lens for the correction of myopia. Eur J Implant Refract Surg. 1989;1:41-3.

3. Dick HB, Alio J, Bianchetti M, et al. Toric phakic intraocular lens: European multicenter study. Ophthalmology. 2003;110(1):150-62.

4. Kohnen T, Cichocki M, Koss MJ. Position of rigid and foldable iris-fixated myopic phakic intraocular lenses evaluated by Scheimpflug photography. J Cataract RefractSurg. 2008;34(1):114-20.

5. Coullet J, Guell JL, Fournie P, et al. Iris-supported phakic lenses (rigid vs foldable version) for treating moderately high myopia: randomized paired eye comparison. Am J Ophthalmol.2006;142(6):909-16.

6. Kohnen T, Shajari M. Ophthalmologe. 2016;113:529. https://doi.org/10.1007/s00347-016-0283-z.

7. Dick HB, Budo C, Malecaze F, et al. Foldable Artiflex phakic intraocular lens for the correction of myopia; two-year follow-up results of a prospective European multicenterstudy. Ophthalmology. 2009;116:671-7.

8. Ruckhofer J, Seyeddain O, Dexl AK, et al. Correction of myopic astigmatism with a foldable iris-claw toric phakic intraocular lens: short-term follow-up. J Cataract Refract Surg. 2012;38(4):582-8.

9. Awadein A, Habib AE. ICL versus Veriflex Phakic IOL for treatment of moderately high myopia: randomized pairedeyecomparison.JRefractSurg.2013;29(7):445-452.https:// doi.org/10.3928/1081597X-20130617-02.

10. Rizk IM, Al-Hessy AA, El-Khouly SE, et al. Visual performance after implantation of two types of phakic foldable intraocular lenses for correction of high myopia. Int J Ophthalmol.2019;12(2):284-90.

11. Menezo JL, Cisneros AL, Rodriguez-Salvador V. Endothelial study of iris-claw phakiclens: four year follow-up.J Cataract RefractSurg. 1998;24(8):1039-49.

12. Ghoreishi M, Masjedi A, Nasrollahi K, et al. Artiflex versus STAAR $^{\circledast}$ implantable contact lenses for correction of high myopia. Oman JOphthalmol.2011;4(3):116-9.

13. Baikoff G, Bourgeon G, Jodai HJ, Fontaine A, Lellis FV, et al. Pigment dispersion and Artisan phakic intraocular lenses: crystalline lens rise as a safety criterion. J Cataract Refract Surg. 2005;31(4):674-80.

14. Menezo JL, Peris-Martínez C, Cisneros AL, et al. Phakic intraocular lenses to correct high myopia: Adatomed, Staar, and Artisan. JCataract Refract Surg. 2004;30(1):33-44.

15. Chen LJ, ChangYJ, KuoJC, etal. Metaanalysis of cataract development after phakic intraocular lens surgery. J Cataract RefractSurg. 2008;34(7):1181-200.

Hinweis des Verlags Der Verlag bleibt in Hinblick auf geografische Zuordnungen und Gebietsbezeichnungen in veröffentlichten Karten und Institutsadressen neutral. 